Marco Bontempi. Marco Bontempi es catedrático de Sociología por la Università di Firenze. Desde hace veinte años lleva estudiando las transformaciones de la participación política y religiosa de los jóvenes, también bajo una óptica comparada, tanto en una perspectiva teórica relacionada con los procesos de secularización y con el debate sobre los paradigmas de modernidad, como por medio de investigaciones empíricas nacionales e internacionales. Sobre estos temas, el autor ha publicado Individualización y transformación de las identidades religiosas y de la cultura política: una perspectiva comparada (2007); Autrement modernes. Jeunes et participation politique au sud de la méditerranée (2008); Biopolitica della giovinezza (2008); Paradigmi di modernità (2008).

Contacto: marco.bontempi@unifi.it 


\section{CONDICIÓN JUVENIL, RELIGIÓN Y MOVILIZACIÓN POLÍTICA EN EL NORTE DE ÁFRICA}

Marco Bontempi

Università degli Studi di Firenze

Fecha de recepción 26 de junio 2014; fecha de aceptación 23 de julio 2014. El artículo que se presenta a continuación se ha desarrollado en el ámbito de un proyecto de investigación promovido por el Dipartimento di Giurisprudenza de la Università degli Studi di Firenze.

\section{Resumen}

En este artículo intento demostrar que el cambio político y religioso que se ha dado en Egipto y en algunos países del Magreb (Marruecos, Túnez) puede interpretarse bajo una perspectiva de modernidad múltiple. El tema central de este artículo es que la modernización política en estos países no sigue el paradigma de la secularización de Occidente basándose en la separación entre religión y Estado. En estos países del Norte de África, los procesos de subjetivización política muestran un enlace muy fuerte con la transformación de la identidad religiosa y el desarrollo de anfiteatros de discusión religiosa, tanto desde un punto de vista pluralista como fundamentalista. Voy a poner en evidencia que la nueva condición juvenil es un factor importante en este cambio. Los jóvenes juegan el papel principal y son los protagonistas de la reislamización de la sociedad, empezada en 1980. La relación muy fuerte entre política y religión aparecida gracias a la primavera árabe no es el resultado de una resistencia tradicionalista y antimoderna, sino que se debe a una multiplicidad de causas que dependen del desarrollo de formas de vida modernas. 


\section{Palabras clave}

Modernidad múltiple, secularización, condición juvenil, socialización religiosa.

\section{Abstract}

In this essay I try to show that the political and religious change happened in Egypt and in some Maghreb countries (Morocco, Tunisia) can be interpreted from a multiple modernities perspective. The central claim of this essay is that the political modernization in these countries doesn't follow the secularization paradigm of the West relying on a separation of religion and state. In these North African countries the processes of political subjectivization show a strong connection to the transformation of religious identity and the development of arenas of religious discussion, both in pluralist that in fundamentalist point of view. I will show that the new condition of youth is an important factor in this change. Young people plays the main role/are key players in the re-Islamization of society, started in the 1980s. The strong connection between politics and religion emerged from the Arab Spring is not the result of a traditionalist and antimodern resistance but it's due to a multiplicity of causes relying on the development of modern forms of life.

\section{Keywords}

Multiple modernities, secularization, youth condition, religious socialization.

En 2001, Eisenstadt originó un debate alrededor de la categoría de multiple modernities, que no deja de ser actual, tanto por la teoría social como por el análisis empírico. Cuando ya no se empieza a pensar en la modernidad como un paradigma universal, sino como una pluralidad de modelos, modernización y occidentalización ya no se pueden considerar procesos isomórficos. Eisenstadt definió el núcleo de la modernidad como la cristalización y la progresión de un específico horizonte cultural que se relaciona con el desarrollo de uno o más conjuntos de formaciones institucionales. Bajo esta óptica, el núcleo conceptual de la categoría de modernidad múltiple se define "in assuming the existence of culturally specific forms of modernity shaped by distinct cultural heritages and sociopolitical conditions. These forms will continue to differ in their value systems, institutions, and other factors". ${ }^{1}$ 
En el desarrollo socio-cultural, el umbral de la modernidad se translimita cuando se empieza a no compartir más la idea por la cual el mundo social es un cosmos organizado por Dios y, por consiguiente, dotado de sentido y éticamente orientado. Poco a poco se pone en marcha una reflexividad que se dirige tanto a los presupuestos existenciales e identitarios como a las condiciones sociales de la legitimación del orden social y político. Una vez alejada de la estabilidad garantizada por la tradición, la relación entre procesos de subjetivización, formaciones institucionales y recursos culturales de legitimidad se abre a procesos incluso muy diferentes. Esta pluralidad de elaboraciones posibles, que fundamenta las formas de vida moderna, por largo rato ha sido balanceada por presuposiciones conceptuales que, poniendo en relación los procesos de modernización de diferentes esferas de vida social, dimensionan el ámbito de las posibles elaboraciones, reduciendo de hecho su variabilidad potencial. Un ejemplo es el enlace entre los procesos de desarrollo democrático y la afirmación de las lógicas de secularización de las subjetividades y de las prácticas sociales. Una importante contribución en la perspectiva de las multiple modernities consiste, precisamente, en la posibilidad de poner en tela de juicio la normatividad de estas presuposiciones a la luz de diferentes posibilidades de articulación de esas conexiones, sin tematizar este cambio de perspectiva como una, diríamos, improbable "salida de la modernidad".

La tesis que subyace a este artículo es que el desarrollo de procesos de individualización y de movilización política en sociedades no europeas -como Egipto o algunos países del Magreb- no funciona siguiendo el clásico paradigma de la secularización -es decir, por medio de procesos de subjetivización en los que se enlazan destradicionalización, racionalismo e individualización y gracias a los cuales se legitima el desarrollo de formas de participación política democrática-, sino traza procesos de individualización y de subjetivización tendencialmente destradicionalizantes, aunque legitimados por transformaciones de la religiosidad a través de las cuales se activan procesos de movilización política y de secularización. Estos cambios se pueden notar empíricamente en una pluralidad de transformaciones de mediano y largo plazo que han caracterizado a las sociedades en cuestión. Claro está, las pocas páginas de un artículo no permiten razonar detenidamente acerca de esta pluralidad. Sin embargo, en esta perspectiva la condición juvenil ofrece un interesante enfoque por medio del cual es posible formular de manera interpretativa una conexión sociológicamente significativa entre algunas variaciones empíricamente detectadas y la creación de formas modernas de la subjetividad y de la movilización política. 


\section{Modernización y transformaciones de la condición juvenil}

Antes que todo, los procesos de modernización se pusieron en marcha en los países MENA, sobre todo a partir de la independencia post-colonial, cuando los Estados nacionales brotaron como factores fundamentales de la regulación social y del crecimiento. El desarrollo de las rentas de hidrocarburos tras el choque petrolero de 1974, junto con el dinero que los emigrados envían a sus familias y a las políticas de ayudas para la lucha contra el analfabetismo y de mejora de las condiciones higiénico-sanitarias, les ha asignado a las instituciones del Estado un papel de absoluta centralidad, fomentando desmedidamente la ocupación por medio del crecimiento del sector público. Las políticas sociales en sociedades con altas tasas de fecundidad han llevado en todos los países del área a la formación de una generación de jóvenes, cuya abundancia numérica es sin precedentes y ya se nota que no se va a reproducir en el futuro. ${ }^{2}$ La actual abundancia numérica de los jóvenes en los países del área es el efecto compuesto de la alta tasa de fecundidad de las madres, que seguía pertinazmente en los años setenta y ochenta del siglo pasado, junto con la fuerte reducción de la mortalidad infantil producida por las políticas sociales y de ayuda, precisamente a partir de la segunda mitad de los años setenta. El aumento de la educación ha afectado mucho a la transformación de la condición juvenil. Por primera vez en la historia de estos países, el acceso en bloque de las chicas a la educación primaria y secundaria ha conllevado consecuencias importantes tanto en la tasa reproductiva, como también en las relaciones de pareja y familiares.

De las inversiones para ampliar la educación puestas en marcha a partir de los años ochenta ha sacado provecho, antes que todo, la generación de los que, hoy día, tienen de 15 a 30 años. El cambio ha sido muy profundo. Sobre la totalidad de los que tienen de 13 a 17 años, la cuota de los matriculados en la escuela secundaria ha pasado en Algeria del 9,6\% de 1971 al 58\% de 1994, al 97,6\% de 2011; en Egipto del 30,4\% de 1971 al 76\% de 1994, al 86,3\% de 2012; en Tunisia del 23\% de 1971 al 53\% de 1994, al 91\% de 2011; en Marruecos del 11\% de 1971 al 37\% de 1994, al 69\% de 2012. Sin embargo, cabe destacar que en Algeria y Tunisia más chicas que chicos continúan estudiando. En 2012, en Tunisia las chicas constituyen el 60\% de los estudiantes universitarios, en Algeria el 59\% y en

2. En los países del Norte de África y del Oriente Medio, ha sido calculado que los que tienen entre 15 y 64 años, es decir, la fuerza de trabajo, han pasado del $40 \%$ de la población en la década 2000-2010, al 80\% a lo largo de los veinte años entre 2000 y 2020. Cfr. R. Assaad, F. Roudi-Fahimi, "Youth in the Middle East and North Africa: Demographic Opportunity or Challenge?”, en Population Reference Bureau, Wahshington D.C., 2007, p. 6. Los demógrafos llaman hoy youth bulge a este aumento de un segmento de la población que, con el paso de las décadas, se desplazará hacia las clases de edades sucesivas, pero que no se volverá a repetir en las generaciones siguientes. 
Egipto el 48\%. Por lo que a este aspecto se refiere, cabe destacar que no hay diferencias con respecto a la orilla norte del Mediterráneo: actualmente, las chicas forman parte del $57 \%$ de los estudiantes universitarios en Italia, el 54\% en España y el 49\% en Grecia ${ }^{3}$.

Sin embargo, estos resultados positivos no pueden disminuir la importancia de la calidad de la instrucción. Algunos estudios ${ }^{4}$ subrayan el problema de la escasa calidad de la educación, atribuyéndola a la persistencia de métodos didácticos basados en el aprendizaje mnemónico y en la falta de estímulos profesionales y económicos para los profesores. También la formación profesional sufre por estos límites y no es útil para facilitar un enlace entre el mercado del trabajo y la formación.

Desde un punto de vista estructural, el mercado del trabajo está dominado por la centralidad del sector público, respecto de un mercado del trabajo privado aún subdimensionado. Las reformas de liberalización del mercado del trabajo, que se han realizado gracias a las instituciones político-económicas internacionales, no han sido beneficiosas para los jóvenes. De hecho, los trabajadores ya empleados, es decir, más adultos, son los que continúan gozando de garantías, mientras que para los jóvenes es mucho más frecuente la experiencia de la precariedad, el empleo con contratos a breve plazo introducidos por las liberalizaciones del mercado del trabajo. A esto se añade una relación negativa entre el nivel de educación y la tasa de desempleo. Los que poseen títulos de estudio más altos sufren un porcentaje de desempleo más elevado. Por varias décadas, el sector público - pues, no el privado- fue él que empleó, de manera casi exclusiva, a los que poseían altos títulos de estudio. Esto ha convertido en menos atractivos a los itinerarios formativos y a las competencias técnico-científicas y profesionales, orientando las elecciones hacia tipos de formación más relacionadas con el mercado del trabajo generado por el desarrollo del welfare. Hoy día, el sistema público encuentra cada vez más dificultades en emplear el creciente número de licenciados, pero la elección del itinerario formativo queda influenciada por el prestigio gozado en el pasado y por la esperanza de un empleo en lo público. Además, esta especie de path dependency es consolidada aún más por la falta de relación entre las competencias adquiridas gracias al sistema de instrucción y aquellas del mercado del trabajo privado.

3. Fuente World Bank, Education Statistics: Core Indicators, http://databank.worldbank.org (26/09/2014).

4. A. Ajbilou, B. Boudarbat, "Youth Exclusion in Morocco: Context, Consequences and Policies", The Middle East Youth Initiative Working Paper n. 5, (Washington and Dubai: Wolfensohn Center for Development and Dubai School of Government, Septiembre de 2007), pp. 13-15, R. Assaad, G. Barsoum, "Youth Exclusion in Egypt: In Search of 'Second Chance”, The Middle East Youth Initiative Working Paper n. 5, (Washington and Dubai: Wolfensohn Center for Development and Dubai School of Government, Septiembre de 2007), p. 25. 
Por lo tanto, se esboza una situación en la que la reducción de las garantías del sector público, la parcial desregulación del sector privado formal y el aumento del trabajo informal ${ }^{5}$ contribuyen a la reducción de las ventajas que el título de estudio puede proporcionar con respecto al mercado del trabajo. Claro está, los que poseen niveles de estudio elevados son, pues, los jóvenes que, en estos años, se hallan experimentando un desempleo de masas y de largo plazo. Además, las razones culturales que caracterizan a la desigualdad de género en el acceso al mercado del trabajo proporcionan el hecho de que, en este panorama, las que sufren las mayores consecuencias son las mujeres, a pesar de que posean un nivel de educación muy a menudo superior al de sus coetáneos chicos. ${ }^{6}$ En este contexto fuertemente caracterizado por condiciones de desigualdad de género, la instrucción constituye la única dimensión en la que las desigualdades de género favorecen a las mujeres, en lugar de los hombres.

Los efectos de esta extraordinaria expansión de la instrucción son muy relevantes desde un punto de vista cultural. De hecho, ponen en marcha procesos de desarrollo de formas modernas de subjetividad. Normalmente esto se da como un proceso de valorización de la autorrealización y de las libertades que respaldan a la autonomía individual, junto con un aumentado interés para la política. Sin embargo, este cambio cultural no encuentra las condiciones de su material concretización por la creciente dificultad -en los últimos tres lustros- de acceder al mundo del trabajo por parte de los jóvenes, en general, y de los jóvenes instruidos, en particular. De ahí que a las modernas formas de subjetivización se oponen no solo las creencias y las prácticas tradicionales, sino también las condiciones materiales que obstaculizan las potencialidades emancipativas de las modernas formas de ocupación generadas por el mercado capitalístico del trabajo. El carácter específico de la actual crisis económica denota que muy difícilmente el mercado capitalístico del trabajo podrá desarrollar en estos países aquella función de integración social y de ayuda material a las formas de emancipación que, en cambio, ha tenido para las mujeres y los jóvenes en la mayoría de los países occidentales. Sin embargo, los efectos de la difusión de la educación y del desarrollo de formas de vida modernas no dejan de producirse tanto en la esfera pública, como en las relaciones privadas. De estas,

5. Desde hace por lo menos una década, en Egipto un número creciente de las entradas de los jóvenes al mundo laboral se realiza en el mercado informal (negro) y es muy alta la dificultad de salir de esta condición para lograr un empleo formal: "casi el 32\% de los trabajos para los nuevos empleados en Egipto en 2005 se ha hallado en el sector informal (...), solo el $11 \%$ de los jóvenes egipcios que buscan su primer trabajo en el sector informal logran obtener un segundo trabajo en el sector formal”. N. Dhillon, T. Yousef (eds.), Generation in Waiting: the Unfulfilled Promise of Young People in the Middle East, Brookings Institution Press, Washington D.C., 2009, p. 22.

6. Al neto de las desigualdades relacionadas con la residencia. De hecho, las diferencias entre áreas rurales y áreas urbanas son muy significativas para el condicionamiento de las posibilidades de acceso al mercado del trabajo, sobre todo para las mujeres. 
pues, vamos a ocuparnos ahora. De hecho, en el ámbito de las relaciones privadas, sobre todo de pareja y familiares, aparecen cambios que muestran lógicas de modernización de las subjetividades que, sin embargo, parecen seguir itinerarios diferentes de los del paradigma de la modernidad occidental.

\section{Las relaciones familiares y de pareja como indicador de los cambios de la subjeti- vidad}

Es notorio que la difusión de la educación conlleva importantes efectos culturales de deslegitimación de las prácticas tradicionales, sobre todo por lo que a la constitución de las identidades relacionadas con los roles sociales se refiere. En las relaciones entre hijos y padres, el bajo nivel de instrucción de los segundos choca con la educación de los primeros, determinando el desarrollo de patentes diferencias intergeneracionales: dentro de la familia, los jóvenes se convierten en los que poseen el saber socialmente legitimado por la sociedad contemporánea y la competencia acerca de las prácticas comunicativas de su circulación a través de los media y de los social networks. Pues bien, se está dando una especie de cambio total en la relación entre edad y conocimiento: del tradicional modelo de la socialización que enlaza el aumento de la edad con la ampliación del conocimiento y que constituye uno de los criterios centrales de la legitimación de la autoridad de los roles de los adultos en las sociedades tradicionales, se pasa a un modelo moderno, en el cual el proceso de socialización ya no tiene su centro exclusivo en la familia y en la estructura tradicional de la autoridad, sino que procede de la combinación de una pluralidad de medios y agencias de socialización dentro de los cuales la familia es uno de ellos, acosado por el aumento de la importancia de la escuela $y$, sobre todo, del grupo de pares. Un importante elemento de novedad deriva del hecho de que esta transformación afecta, en igual medida, tanto a los chicos como a las chicas, repercutiendo profundamente en la estructura jerárquica del sistema patriarcal y desquiciando las dos líneas de subalterneidad que lo estructuran: la de género y la de las clases de edad. En la primera, la mujer está sometida al hombre, en la segunda los hermanos menores al mayor, es decir, al que se convertirá en el futuro patriarca. Si, por una parte, el aumento de la instrucción entre las mujeres aleja la edad del matrimonio y legitima la aspiración a trabajar, por lo menos hasta el matrimonio o al nacimiento del primer hijo, por otra parte, erosiona desde la base la lógica asimétrica del modelo patriarcal de las relaciones familiares pero -tal como veremos ahora- no registra la salida de una socialización marcadamente gendered. Pero claro, no nos encontramos ante una emancipación en el sentido occidental del término. En cambio, se da un proceso dife- 
rente, en el que la tradición no es rechazada, sino reelaborada junto con el desarrollo de formas de modernización. Los cambios de la condición juvenil no modelan la forma del conflicto entre las generaciones, pero -desarrollándose en sociedades procedentes de un proceso de "modernización desde lo alto"- esbozan un modelo de modernidad en el cual los elementos de innovación están relacionados con rasgos culturales tradicionales en varios modos, tanto por lo que respecta a la posición social, como a las formas de capital cultural a las que se refiere.

En esta perspectiva, algunas investigaciones sobre la dinámica de las relaciones intergeneracionales parecen ser sobremanera interesantes. En particular, los resultados de un estudio empírico llevado a cabo en 1999 en Egipto sobre una muestra representativa nacional de jóvenes ${ }^{7}$ evidenciaba que, ya hace quince años, el 68\% de los chicos y el 61\% de las chicas entre 16 y 19 años tenían un nivel de instrucción más elevado respecto de su padre. Mayor aún es la cuota de los que tienen un nivel de instrucción superior al de su madre ( $80 \%$ de los chicos y $74 \%$ de las chicas). Esta transformación favorece la deslegitimación de las condiciones de la autoridad masculina en las relaciones de pareja. Los jóvenes entrevistados han declarado -con diferencias entre chicos y chicas- una orientación en su mayoría favorable a la paridad de instrucción entre hombre y mujer (el $48 \%$ de los chicos y el 67\% de las chicas) y -de manera aún más fuerte-a una diferencia de edad en la pareja de no más de 5 años (el 94\% de los chicos y el $86 \%$ de las chicas), y esto destaca aún más en un contexto en el que, entre los adultos, solo una pareja casada de cada cinco tiene el mismo nivel de instrucción, mientras que en poco más de la mitad de las parejas casadas se halla una diferencia de edad de más de 5 años.

Sin embargo, frente a posiciones modernizantes, parecen sobremanera interesantes los datos relativos a dos indicadores de la subalterneidad de la mujer respecto del hombre. El primer indicador muestra que, en 1999, una minoría de jóvenes -el 26\% de los chicos y el 16\% de las chicas- apoyaba la tradicional práctica que obliga la mujer a casarse con un pariente si este lo pide y si el padre está de acuerdo. Esto ocurre a raíz del hecho de que el matrimonio de jóvenes entre parientes, en Egipto, es una práctica bastante difundida, aunque no mayoritaria. El más reciente Survey on Young People in Egypt 2010, relativo a una muestra nacional representativa de los jóvenes de 10 a 29 años, indica que el 34\% de los matrimonios de los jóvenes sigue dándose entre parientes. ${ }^{8}$

El segundo indicador atañe a la práctica de la escisión de las chicas antes del matri-

7. S. Al Tawila, H. Wassef, B. Ibrahim, "Changement social et dynamique adolescents-parents en Egypte”, en Monde arabe. Maghreb-Machrek, n.171-172, Enero-Junio de 2001, pp. 52-66.

8. Population Council, Survey of Young People in Egypt, Final Report, Population Council, West Asia and North Africa Office, Cairo, 2010, p. 17. 
monio. Se trata, pues, de una práctica extremadamente difundida en la sociedad egipcia, con escasas diferencias de pensamiento entre jóvenes y adultos. Los jóvenes entrevistados en 1999 se declararon de acuerdo con esta práctica con proporciones muy altas: el 56\% de las chicas y el 76\% de los chicos. El Survey on Young People in Egypt 2010 muestra que el $82 \%$ de las chicas de 10 a 29 años es circuncisa, pero también que esta práctica -que, tradicionalmente, se impone a las niñas a los 9 años - parece estar bajando si se considera que ha sido circuncidado el $92 \%$ de las chicas entre 22 y 29 años -el segmento más adulto-, pero ‘solo’ el 66\% de las entre 10 y 14 años, es decir, el segmento más joven. ${ }^{9}$

En fin, ambos estudios confirman una marcada división sexual de los roles en los quehaceres domésticos. En este caso, la orientación de los jóvenes coincide, pues, con la de los adultos; de hecho, casi todos los jóvenes otorgan exclusivamente al hombre la tarea de proveer económicamente a la familia, mientras que considera específicamente femenina la tarea del cuidado de los hijos.

Por lo tanto, la elaboración de la subjetividad juvenil sigue líneas diferenciadas dependiendo del sexo; en particular, los chicos aparecen comprometidos más que las chicas en la reproducción de roles e identidades basados en la tradición. Por su parte, las chicas experimentan condiciones ambivalentes de subjetivización, por una parte de subalterneidad según formas tradicionales, por otra parte de desarrollo de relaciones paritarias con la pareja y de orientaciones a la autonomía individual. La propensión de las chicas a preferir más que los chicos papeles e identidades modernas se conjuga con la adhesión a modelos e identidades tradicionales, tal como la aceptación del destino femenino dirigido al cuidado de los hijos antes que al trabajo y, aún más, con el lento alejamiento de la práctica de la escisión para sus hijas. Sin embargo, en términos generacionales, los jóvenes marcan la distancia de los adultos por lo que a las cuestiones relacionadas con la esfera de la subjetividad y de la comunicación en la pareja se refiere, declarándose favorables a niveles similares de instrucción y de edad, mientras que por lo que atañe a la esfera de los roles, muestran una distancia menor de los adultos a la hora de compartir inclinaciones tradicionales. Por lo tanto, las formas de la subjetividad juvenil parecen estar caracterizadas por una variedad de dimensiones en las que tradición y modernidad se enlazan, delineando una trama peculiar De los procesos de los que, hasta ahora, hemos hablado brevemente, cabe subrayar dos aspectos: el primero es la pluralización de las agencias y de los canales de socialización que, a la hora de

9. Ibid., p. 8 . 
limitar la influencia de la familia y de incrementar el papel de otras agencias como la escuela y el grupo de pares, contribuye al desarrollo de formas de subjetividad estructuradas en la negociación de los valores y de las identidades. Se trata de un cambio que puede ser considerado irreversible, dada la complejidad de las condiciones que lo han posibilitado. El segundo aspecto se relaciona con la marcada connotación de género a la hora de definir los papeles y las identidades a ellos vinculados. Si, por un lado, se debe entender como una persistencia de las formas tradicionales, por otro lado, constituye también un ámbito en el cual se hace cada vez más intenso el debate en la esfera pública. Precisamente en esta se halla otra dimensión fundamental de estos peculiares procesos de modernización: la importancia de la religión como recurso de legitimidad en los debates acerca de la afirmación de las subjetividades modernas. Los resultados de algunas investigaciones sobre la socialización religiosa en Marruecos son particularmente interesantes para nuestro análisis.

\section{Socialización y procesos de modernización}

Cualquier estudio acerca de la socialización religiosa de los jóvenes musulmanes, tanto en Marruecos como en otros países musulmanes, en primer lugar, debe considerar que, a partir de los últimos veinte años del siglo XX, el interés y la adhesión al islam han registrado un incremento consistente y paralelo aldesarrollo de los procesos de modernización. En muchos países musulmanes, esta creciente importancia de la religión se ha combinado con un proceso de transformación de la religiosidad que ha caracterizado a las formas de vida cotidiana y del que precisamente los jóvenes han sido los protagonistas. Se trata de un cambio muy significativo, tanto por lo que a las secuelas sociales que conlleva se refiere, como porque pone en seria tela de juicio el carácter paradigmático de la teoría de la modernidad occidental que descubre en la secularización de la vida cotidiana una de las condiciones más importantes de los procesos de destradicionalización de la cultura. De hecho, la reislamización de la vida cotidiana no se configura como una resistencia a la modernidad en nombre de valores tradicionales, sino como el comienzo de un proceso que fluctúa entre dos polaridades -que podríamos sintetizar como la “islamización de la modernidad” y la "modernización del Islam"- y que confiere un papel central a la legitimación religiosa de los procesos de subjetivización modernos. Bajo esta óptica, hay que destacar que al inicio de la modernización -en los años sesenta y setenta- la práctica religiosa de los jóvenes se había reducido progresivamente, hasta 
encasillarse en dimensiones muy limitadas. A partir de los años ochenta, pues, se asiste a una reactivación que marca una acentuada discontinuidad respecto de las prácticas y de la religiosidad del pasado y de las generaciones adultas. Tres estudios -que se llevaron a cabo entre 1983 y 1996- explican bien esta transición (tabla. 1). Más allá de las diferencias de detalle, de los datos se desprende una especie de cambio total en el cual la práctica de rezar con regularidad pasa de ser menos de un décimo de la muestra, en 1983, a alrededor de la mitad de la muestra en la década siguiente y, viceversa, los que declaran que no rezan nunca, con respecto a casi la mitad de la muestra, se reducen a un décimo.

Tabla. 1 Tres estudios sobre estudiantes de secundaria y universitarios (\%)

\begin{tabular}{|l|c|c|c|}
\hline & $\begin{array}{c}\text { No reza } \\
\text { nunca }\end{array}$ & $\begin{array}{c}\text { Reza con } \\
\text { regularidad }\end{array}$ & $\begin{array}{c}\text { Reza de } \\
\text { manera } \\
\text { esporádica }\end{array}$ \\
\hline Casablanca, 1983 & 48 & 8 & 34 \\
\hline Rabat, 1993 & 17 & 54 & 27 \\
\hline Rabat, 1996 & 9 & 45 & 46 \\
\hline
\end{tabular}

Fuente: Bourquia-El Ayadi-El Harras-Rachik (2000, 14-18).

¿Cómo se realiza la socialización religiosa de los jóvenes? ¿Cuáles son los principales canales de socialización? Tales aspectos son significativos para comprender la relación entre reislamización y modernización. Varios estudios ponen de manifiesto la relevancia de cuatro vectores de la socialización religiosa.

El primero está constituido por la familia y, sobre todo, por la educación religiosa que la madre imparte a los hijos: "analfabeta en la mayoría de los casos, la madre comunica a sus hijos un islam que hace hincapié en la fe, en el fatalismo y en el hecho de creer en el destino. En general, no teniendo las mujeres acceso a los textos, desarrollan una relación estrecha con las formas populares de lo sagrado".${ }^{10}$ En muchos casos, pues, el islam enseñado en familia posee marcadas connotaciones de religión popular, ya que la familia es el canal de socialización que más está involucrado en las prácticas tradicio-

10. R. Bourquia, M. El Ayadi, M. El Harras, H. Rachik, Les jeunes et les valeurs religieuses, Editions EDDIF - M.Y.B. Retnani, Casablanca, 2000, pp. 21-22. 
nales. Tales formas están disminuyendo fuertemente entre los jóvenes: por ejemplo, un estudio de $2003^{11}$ demuestra que la creencia en los talismanes afecta solo al $8 \%$ de los que tienen de 20 a 30 años, pero al 36\% de los que tienen más de 60 años; también la creencia/culto en los santos (presente solo en el islam popular) se diferencia marcadamente entre los jóvenes y los mayores: de los que tienen entre 20 y 30 años solo el 10\% está a favor, mientras que entre los que tienen más de sesenta años lo está el 56\%. Este mismo estudio demuestra también la disminución de la asistencia a las escuelas coránicas - centro fundamental de la formación religiosa- por parte de los jóvenes: en 2003, fueron cursadas por el $28 \%$ de los que tienen menos de 20 años y por el $38 \%$ de los que tienen entre 20 y 30 años, respecto del $48 \%$ de los que tienen más de cincuenta años.

El segundo vector de la socialización religiosa está representado por la escuela. Los jóvenes aprenden en la escuela el islam ortodoxo gracias a la lectura y conocimiento del Corán y a sus interpretaciones consolidadas. En los libros escolares, la referencia alislam también sirve para legitimar el orden social y cultural, entrelazándolo con el estudio de las asignaturas humanísticas ${ }^{12}$.

El tercer vector de la socialización religiosa es el grupo de pares. En los procesos de reislamización, las relaciones entre pares ofrecen un importante contexto de debate acerca de las conexiones entre islam y formas modernas de vida y, a menudo, es en esos contextos que se forman las elecciones individuales en favor de una u otra corriente del islam: "Es a este nivel que se desarrolla la socialización islámica, que se asienta en las adquisiciones de la socialización religiosa para imponerle una nueva definición. Esta esboza unas líneas de diferenciación dentro de la esfera de la religiosidad. De esta manera emergen grupos que llevan a cabo interpretaciones diferentes de la religión". ${ }^{13}$ Las asociaciones islámicas (dentro de las que la más conocida es la de los Hermanos Musulmanes), muy presentes en los barrios urbanos, en las mezquitas, en las escuelas y en las universidades, ofrecen una forma organizada de compromiso religioso que excede a aquellas tradicionales, tanto por el reconocimiento de la importancia del debate acerca de temas de práctica y vida religiosa por parte de loscreyentes, como por la posibilidad de formas de compromiso religiosamente calificados en actividades de voluntariado social. Esta doble connotación de ámbito de debate y de acción -ambos religiosamente calificados, por medio de los cuales se elaboran formas de alejamiento de roles y prácti-

11. M. Ababou, "The Impact of Age, Generation and Sex Variables on Religious Beliefs and Practices in Morocco", en Social Compass, 52, 2005, pp. 31-44.

12. Para un interesante análisis acerca de la lógica argumentativa religiosa en los libros escolares en Marruecos, cfr. R. Bourquia, M. El Ayadi, M. El Harras, H. Rachik, Les jeunes et les valeurs religieuses,pp. 157-165.

13. Ibid., pp. 23-24. 
cas del islam tradicional- fundamenta la difusión y el asentimiento popular hacia esas asociaciones. Asimismo, ya mucho antes de las “primaveras árabes” ha sido subrayado que henceforth, the religious accompaniment of Moroccan youths will be carried out by this type of association, which is well established. This religious socialization is not only based on an oral tradition but also on a written means, by the distribution of books (free or inexpensive), as well as by new means of communication (the Internet, video cassettes, audio cassettes, etc. $){ }^{14}$

Por lo tanto, bajo esta óptica, los medios de comunicación constituyen el cuarto vector de la socialización religiosa. En los nuevos media han sido acogidos los temas y los significados religiosos, reconsiderados de manera crítica tanto respecto de la política y de la cultura occidental, como respecto de las prácticas, roles y formas del islam tradicional. La comunicación digital -sobre todo los predicadores en los canales satelitales y en los social networks en internet-representa la última forma de este proceso empezado en los años ochenta y noventa con audio y videocasetes y con la industria editorial religiosa de masa. ${ }^{15}$

En síntesis, estos cuatro vectores esbozan tres lógicas sociológicamente distintas que están en marcha en los procesos de socialización religiosa de los jóvenes marroquíes:

a) socialización religiosa tradicional-popular desarrollada en familia con el papel fundamental de la madre (culto de los santos, de las tumbas de los santos, talismanes, amuletos, prácticas mágicas con el Corán, etc.); b) socialización religioso-nacional puesta en marcha por la escuela. Este canal representa una mezcla de islam ortodoxo y de identidad nacional que halla su propia representación en los libros de texto controlados por el Estado; c) socialización religiosa de la religiosidad discutida y elegida por el individuo, cuyos factores principales son la difusión de elevados niveles de instrucción entre los jóvenes, la importancia de las relaciones entre pares, las asociaciones islámicas y la difusión de las formas de comunicación digital.

Así que los procesos de modernización cultural parecen esbozar un itinerario de transformación en el que la religión actúa como fuente de legitimidad de los procesos de autonomización del individuo y de afirmación de las formas de subjetivización

\footnotetext{
14. M. Ababou, "The Impact of Age, Generation and Sex Variables on Religious Beliefs and Practices in Morocco”, p. 38. 15. R. Hadj-Moussa, "Lier ou délier? Les jeunes Maghrébins au-delà de la Méditerranée”, Relación presentada en el IV Congresso Internazionale Young people and Society, Forlì 26-28 Marzo de 2009, pp. 9-10: "El atractivo que ejercen los canales satelitares está atestiguado por la notable presencia de la dimensión religiosa. (...) Hay que destacar que estos canales no hacen otra cosa sino acentuar un movimiento que ya existía antes gracias a los antiguos medios como los audiocasetes y videocasetes y el mundo de la industria editorial. Los programas religiosos cosechan cada vez más espectadores, sobre todo durante el mes de Ramadán. (...) De hecho, las personalidades religiosas o los nuevos predicadores movilizan un público extremadamente variado y numeroso. (...) Sin embargo, estas nuevas formas de religiosidad que aparecen como procesos de reislamización son fenómenos muy inestables, en continua redefinición".
} 
ancladas a valores de autorrealización. Procesos diferentes como las transformaciones demográficas, la difusión de la instrucción, los cambios en las relaciones familiares y de pareja y la transformación de los vectores de la socialización coinciden en delinear condiciones de modernización, protagonizadas por las nuevas generaciones, que difieren del paradigma clásico de la modernización por medio de la secularización; condiciones que, por muchas razones, se deben considerar consecuencias de transformaciones culturales no solo irreversibles, sino también en expansión. Como se puede notar, se trata de un cambio nada claro y conflictual, pero lo que vale la pena subrayar, antes que todo, es el comienzo de debates sobre issues religiosas y socio-religiosas puestos en marcha por las transformaciones de la modernidad como, por ejemplo, las discusiones sobre roles, formas y espacios de la autodeterminación femenina. Ya en 2004, Pippa Norris y Ronald Inglehart, en Sacred and Secular. Religion and Politics Worldwide -un estudio comparativo internacional basado en las World Values Surveys-subrayaban cómo

el Occidente es mucho más igualitario y liberal que todas las demás sociedades, sobre todo que las musulmanas. (...) Sin embargo, cabe destacar que cualquier tesis que hable de un choque de civilizaciones o que, en particular, sostenga que las sociedades islámicas y occidentales poseen valores políticos fundamentalmente diferentes, ejemplifica de manera inaceptable la evidencia empírica. Las sociedades islámicas y occidentales comparten una orientación positiva hacia los ideales democráticos. (...) En los países islámicos existe un espantoso consenso hacia la democracia, también en aquellos con regímenes autoritarios. El decisivo punto de ruptura entre Occidente e Islam no atañe a la democracia, sino a cuestiones de igualdad de género y de libertad sexual. ${ }^{16}$

Lejos de querer ejemplificar demasiado un conjunto de procesos complejos, igual es posible sostener que se ha empezado a debatir antes en las tertulias religiosas que en las "primaveras árabes". Claro está, esto no excluye las radicalizaciones desarrolladas por algunas formas de fundamentalismo, sino conlleva una complejidad a la hora de analizar las relaciones entre religión, sociedad y política que no puede ser desatendida. Desde este punto de vista, los temas y las formas de la movilización política de las "primaveras árabes" muestran una importante convergencia con los procesos antes descritos. En

16. R. Inglehart, P. Norris, Sacred and Secular: Reexamining The Secularization Thesis, Cambridge University Press, Cambridge, 2004. 
los párrafos que se presentan continuación intentaremos señalar estas convergencias, reconstruyendo algunos elementos de interpretación de estas movilizaciones explicados por Olivier Roy.

\section{Islamismo, movilización política y lógicas de secularización}

Al principio, las primaveras árabes no tuvieron las características de una revolución para la afirmación de un estado islámico, ni aquellas de una revolución nacionalista árabe. Las peticiones iniciales atañían a la democracia, dignidad, elecciones, respeto de los derechos humanos y, sobre todo, a los derechos de ciudadanía de los individuos, dejando de lado a entidades simbólico-colectivas como el pueblo, la umma, la nación árabe. No se ha tratado de un movimiento revolucionario en el sentido clásico del término: los manifestantes no se han apoderado del poder de manera violenta, ni tampoco lo han intentado. Simplemente, lo que querían era trazar un nuevo escenario político.

La tesis de Olivier Roy es que, a pesar del golpe en Egipto y del florecimiento del terrorismo islámico en Iraq y otros lugares, el proceso de llegada al poder de los partidos islámicos de manera democrática es mucho más amplio (Tunisia, Turquía, Marruecos, Jordania) y marca un cambio muy significativo de inicio de un proceso de democratización en las sociedades árabes. Un proceso muy contrastado y expuesto a detenimientos, distorsiones, más sociológicamente atribuible a aquellos cambios sociales y culturales de los que hemos hablado antes, cuyos efectos influyen en la relación entre política y sociedad. Esto permite que la democratización no se realice de pronto y por medio del sistema político, afectando a los actores políticos con resultados de convergencia. Se trata de un proceso social que es, antes que todo, democratización en la manera de vivir la religiosidad y de considerar -en relación con los cambios en esta- el papel de la religión en la vida social y las relaciones entre religión y política. Una transformación que no atañe a las doctrinas, sino a la acción política cotidiana.

La individualización que la democracia presupone en los países musulmanes se legitima por medio de la religión o, mejor dicho, a través de una transformación de la religiosidad, de tradicional a moderna, que legitima la individualización pero en un ámbito de relevancia de lo sagrado en la vida colectiva. Esto no excluye la secularización, sino que configura procesos de la misma promocionados por la transformación de la religiosidad, no alternativos a la religión. 
Bajo esta perspectiva, Roy observa que "los actores religiosos fundamentalistas como los islámicos de Tunisia o incluso los Salafitas de Egipto podrían convertirse en actores reacios de una forma de secularización específicamente política que no se debería confundir de ninguna manera con una secularización de la sociedad" ${ }^{17}$ Pues, actores, porque solo con los cambios democráticos generados por las primaveras árabes los partidos políticos islámicos han podido entrar en los sistemas políticos. Reacios porque el núcleo central de la agenda de estos partidos es religioso y su plantilla política se relaciona con la acción política cotidiana como si fuera una realidad diferente de la religión. De una secularización específicamente política, porque la legitimación religiosa de la individualización marca la entrada de las libertades individuales en la política. De tal manera que la política se convierte en un campo de batalla y competición que, para los islámicos, se debe dirigir a las libertades individuales, reconociéndolas a la luz de los valores religiosos, antes que todo como posibilidad de interpretaciones variadas en el interior del campo religioso, llevadas a cabo también por actores no calificados -como los simples creyentes o los predicadores- quienes, en el Islam tradicional, no tienen este espacio de autonomía de los intérpretes calificados, como los ulemas. En resumidas cuentas, a través de este cambio de la religiosidad, los temas de la autonomía del individuo, de las relaciones entre los géneros y de las diversidades de opinión entran en la política por medio de los valores religiosos. Esto no significa que los religiosos ya son democráticos en política, sino que la traducción en forma política del consenso que poseen en la sociedad a través de elecciones democráticas los empuja hacia la aceptación de la pluralidad de los puntos de vista -antes que todo religiosos- sobre la sociedad y sobre las formas de la regulación política de la sociedad. Esto permite el hecho de que la sociedad no se secularice, porque es la religión la que desarrolla un papel relevante pero, al mismo tiempo, la política es aceptada como una esfera cotidiana de acción caracterizada por lógicas propias que son reconocidas en la acción de gobierno, por ejemplo, a la hora de admitir que las políticas económicas deben tener en cuenta lógicas no subordinadas a los principios religiosos y también a la hora de aceptar y defender las instituciones políticas democráticas como espacio común a islámicos y no islámicos. Pues bien, secularización política significa reconocer a las instituciones políticas una lógica propia, que puede ser inspirada por valores religiosos, aunque sea diferente del ámbito religioso; de hecho, Roy subraya la importancia de tener separadas, tanto desde un punto de vista conceptual como desde

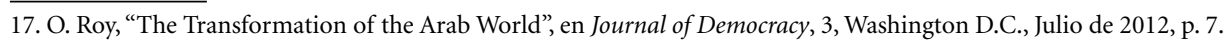


un punto de vista fenomenológico, la fe y la identidad religiosa. La "identidad islámica" de la que se ha hablado mucho justo después de las primaveras árabes

no significa que las mezquitas, de ahora en adelante, estarán más atestadas. En política, identidad religiosa y fe son dos conceptos diferentes (y, quizá, opuestos). La identidad puede ser una manera para esconder la fe debajo de la actuación política laica. Los islamistas, como los salafitas, están entrando en un espacio caracterizado por determinados vínculos que no solo limitarán su supuesta "agenda escondida" de instituir un estado islámico, sino también los empujarán hacia una manera de gobernar más abierta y democrática, siendo esta su única posibilidad de quedar actuando en vida política. De tal forma que los islamistas, y hasta los salafitas, llegarán a ser agentes reacios de democratización. ${ }^{18}$

\section{Las consecuencias secularizantes del fundamentalismo}

Es la individualización de la religiosidad la que ha favorecido y llevado, en los últimos treinta años, a la centralidad de la religión en la vida cotidiana. En la concepción occidental de la modernidad, este dato de hecho parece extraño o hasta inadmisible. En efecto, la centralidad de la religión en la vida cotidiana a menudo se asocia con las sociedades tradicionales y no con la individualización de la religiosidad. ${ }^{19}$ Esta doble dinámica de individualización, expansión y cambio de la religiosidad ha llevado al desarrollo de una diversificación de los movimientos religiosos. En las décadas pasadas, justo los movimientos religiosos conservadores, como los salafitas, han sido apoyados y destituidos por regímenes autoritario-dictatoriales, deseosos de reducir la atractividad de los MB, y esto ha pasado porque la centralidad que confieren a la religión los hacía considerar menos sensibles a la politicización de sus temas y, pues, preferidos por los regímenes autoritarios. Esto significa, antes que todo, que en los países en donde los islamistas han tenido un éxito electoral justo el desarrollo de la centralidad de la religión en la vida cotidiana ha hecho posible que un solo partido o movimiento religioso haya podido arrogarse el derecho de hablar por cuenta del islam, tal como ha ocurrido, pues, en la revolución khomeinista. Esta pluralización no afecta a los cimientos teológicos

18. Ibid., p. 8.

19. Eso pasa solo en la reconstrucción weberiana del nexo religión-actuación económica, aunque el mismo Weber lo haya considerado como un fenómeno de breve duración y deshomogéneamente distribuido. 
del islam, más bien se sitúa en el plano de la religiosidad práctica en la vida cotidiana. Como es notorio, esto puede llevar al surgimiento de numerosos contrastes sobre las interpretaciones y las formas y grados de aplicación de las normas religiosas. Por ejemplo, ¿cómo realizar los principios del islam en la acción política? ¿Cuáles leyes pueden ser adaptadas al islam? ¿Quién lo establece? ¿Cómo?

Inevitablemente, las diversas posiciones entre salafitas e islamistas generan un debate que afecta también a la comunidad de los creyentes. Por ejemplo, en el pasado, los salafitas se negaron a formar partidos porque contraponían la democracia a la centralidad del islam. Luego, con los cambios puestos en marcha por las primaveras árabes, en Egipto y con un partido de reciente formación, ellos entraron en competición electoral con los Hermanos Musulmanes. Roy observa que este pasaje es ya una forma de secularización porque los lleva en el interior de las dinámicas de la lucha política democrática, aunque con el objetivo de afirmar la shari'a, más que la democracia. Sin embargo, la búsqueda del consenso electoral llevará aún más a una connotación post-ideológica del islamismo, con la finalidad de ampliar el consenso electoral más allá del núcleo duro de los electores religiosos, incluyendo al electorado conservador de las clases medias que encuentra en la evocación de los valores tradicionales -como en el caso de la shari'auna especie de punto de referencia respecto de una sociedad en profunda transformación. A un observador no implicado en el asunto todo eso no parece serle tan diferente del uso que ciertos partidos conservadores europeos hacen del cristianismo en función político-electoral.

En estas transformaciones post-ideológicas, el islam adquiere el papel de horizonte cultural, de un conjunto de valores adquiridos como identidad cultural antes que solo como vínculos de acatamiento de prácticas y significados trasmitidos tradicionalmente. Esto cambia el estatuto del creyente y las formas de la religiosidad, y eso ocurre sin modificar el núcleo doctrinal y las diferencias "confesionales" que se le pueden atribuir. Antes que todo, se trata de un cambio de la religiosidad que resiente y se refleja en varias dimensiones de la transformación social, pero no es inmediatamente una secularización, porque no conlleva una disminución de la relevancia de la religión en su relación con la política.

Estos cambios esbozan una transformación que Asef Bayat ha llamado post-islamis$m o$, lo cual no significa la desaparición de los islamistas, sino indica un proceso en el cual se ha ido delineando cada vez más la falta de correspondencia entre las utopías islamistas y las realidades sociales de los países islámicos. Las transformaciones generadas por los procesos de modernización han favorecido más la individualización que la 
creación de instituciones económicas, sociales, culturales y políticas islámicas. En otras palabras, el pasaje al post-islamismo marca el final de las ideologías -en un contexto en el que la religión sigue teniendo un importante papel de legitimación social- y también de la individualización.

Es importante subrayar que el revival religioso en el islam no se debe identificar con el islamismo; pues sí, ha convertido a los islamistas en un tipo de actores religiosos entre otros, diferenciando el campo religioso. Los islamistas no han sido los que han puesto en marcha las primaveras árabes, a veces han sacado provecho de ellas en medida superior a lo esperado y este consenso los ha dificultado a la hora de transformarse en sentido democrático, habiéndose alejado de las formas radicales del fundamentalismo violento. En síntesis, "no son, pues, ni laicistas, ni liberales, pero pueden ser democráticos". ${ }^{20}$ Aunque con innumerables cambios y diferencias, lo que puede ocurrir es el desarrollo de una creciente autonomía del debate religioso del control político e ideológico, tanto respecto de los gobiernos y de los Estados, como respecto de la pretensión de aspirar a un monopolio del poder por parte de un partido o movimiento político religiosamente fundamentalista. El éxito de la difusión del islam en la vida cotidiana ha diversificado el campo del islamismo, facilitando el desarrollo de debates y la necesidad de alianzas para poder tener alguna influencia en la sociedad. Se trata, pues, de una nueva forma de secularismo político en el que

la religión ya no decide cómo la política debería ser, pero se medirá con las acciones políticas. Lo que está en juego es la reformulación del lugar de la religión en la esfera pública (...) En vez de la secularización de la sociedad, sería mejor si habláramos de "autonomización" de la política respecto de la religión y de la religión respecto de la política, debida a la diversificación del campo religioso y de la imposibilidad de reconstruir la religión como una ideología política. ${ }^{21}$

Concluyendo, lo que fundamenta estos cambios políticos es la convergencia entre procesos de cambio social y cultural de largo plazo y transformaciones de la religiosidad, sobre todo en las jóvenes generaciones, y esto proporciona el hecho de que lo que, en Occidente, se percibe como un retorno a la tradición, de lo contrario es

20. O. Roy, "The Transformation of the Arab World", p. 14.

21. Ibid., pp. 17-18. 
un profundo cambio del islam tradicional, cambio que está abriendo camino a un ámbito religioso más abierto y diversificado. Igual que la Reforma Protestante que, a pesar de la aspiración a un regreso a las escrituras, sin quererlo abrió las puertas a las formas modernas de religiosidad, el islamismo está abriendo la puerta a nuevas formas de religiosidad por medio de su pasaje a la política. Además, tanto como instrumento como consecuencia de la deculturación del islam, el fundamentalismo ha ayudado al islam a introducirse en el mercado religioso global. ${ }^{22}$

Traducción del italiano de M. Colucciello

22. O. Roy, “There will be no Islamist Revolution”, en Journal of Democracy, 1, Washington D.C., Enero de 2013, p. 19. 